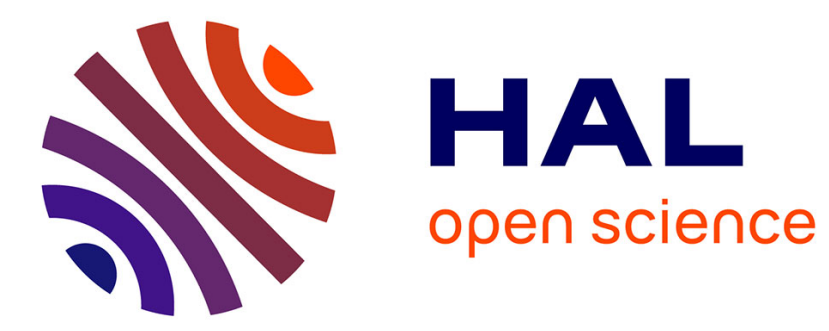

\title{
Le marquage du nombre dans le parler occitan des Ramats (TO, Italie)
}

Jean Sibille

\section{To cite this version:}

Jean Sibille. Le marquage du nombre dans le parler occitan des Ramats (TO, Italie). Zeitschrift für romanische Philologie, 2013, 129 (3), pp.629-651. hal-00952238

\section{HAL Id: hal-00952238 \\ https://hal-univ-tlse2.archives-ouvertes.fr/hal-00952238}

Submitted on 4 Apr 2016

HAL is a multi-disciplinary open access archive for the deposit and dissemination of scientific research documents, whether they are published or not. The documents may come from teaching and research institutions in France or abroad, or from public or private research centers.
L'archive ouverte pluridisciplinaire HAL, est destinée au dépôt et à la diffusion de documents scientifiques de niveau recherche, publiés ou non, émanant des établissements d'enseignement et de recherche français ou étrangers, des laboratoires publics ou privés. 


\title{
Le marquage du nombre \\ dans le parler occitan des Ramats (TO, Italie)
}

\author{
Jean SIBILLE, \\ CLLE-ERSS (UMR 5263) \\ Université de Toulouse II -Le Mirail
}

Dans les langues romanes standard, les systèmes de marquage du pluriel sont relativement simples. En revanche, certaines variétés romanes vernaculaires possèdent des systèmes complexes faisant appel, dans une même variété, à plusieurs procédés de marquage de natures différentes. Dans la variété étudiée, les substantifs se répartissent en dix classes : une classe de substantifs invariables et neuf classes dans lesquelles le pluriel peut être marqué : par un suffixe vocalique alternant (/v'a so/ 'vache', pl. /v'a $\int \varepsilon /$ ), par allongement vocalique, ou par

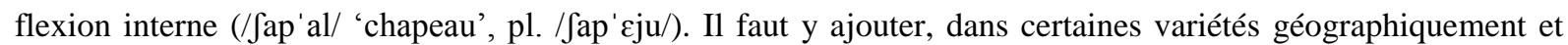
linguistiquement proches, le marquage par un morphème -/s/ agglutiné au radical. L'étude des systèmes de marquage du pluriel dans différentes variétés romanes non standard, pourrait contribuer à une meilleure compréhension de la façon dont interagissent les contraintes phonologiques, morphologiques et syntaxiques.

Jean Sibille, Le marquage du nombre dans le parler occitan des Ramats (TO, Italie), $\mathrm{ZrP}, \mathrm{n}^{\circ} 129-3$; pp. 629-651

\section{Introduction}

Si l'on met à part le roumain qui compte un nombre important de pluriels non prévisibles, dans les langues romanes standard, les systèmes de marquage du pluriel des noms et des adjectifs, sont relativement simples.

L'espagnol utilise une marque $-s$ ou -es, agglutinée à la forme du singulier, la marque du pluriel y est strictement concaténatoire : gato 'chat', gatos 'chats'; casa 'maison', casas 'maisons', árbol 'arbre', árboles 'arbres'. Dans ce système, le singulier est la forme non marquée, le pluriel la forme marquée ${ }^{1}$. Le portugais possède un système comparable, avec toutefois, modification du radical pour les substantifs terminés par $/ \mathrm{m} /$ ou $/ 1 /$, et certains substantifs terminés par une diphtongue nasale: homem 'homme', homens 'hommes'; avantal 'tablier', avantais 'tabliers' ; cão 'chien', cães 'chiens'...

En italien on a un système de suffixes vocaliques alternant en fonction du nombre et variables suivant les différentes classes nominales : $1 /$ masc. sing. $-o$, pl. $-i:$ gatto 'chat', gatti 'chats' ; $2 /$ masc. sing. - $-a$, pl. - $i$ : autista 'chauffeur', autisti 'chauffeurs'; 3/ fém. sing. - $a$, pl. - : casa 'maison', case 'maisons'; 4/ masc. ou fém., sing. -e, pl. - $i$ : prete 'prêtre' (m.), preti 'prêtres' ; parte 'partie' (f.), parti 'parties' ${ }^{2}$. Il existe également une classe de substantifs oxytons qui sont invariables: virtù, servitù, lunedì... et les dérivés en -tà (libertà, semplicità...), ainsi que quelques pluriels irréguliers : uomo 'homme', uomini 'hommes'; bue 'bœuf', buoi 'bœufs'... Les emprunts à des langues étrangères, non intégrés ou incomplètement intégrés, sont également invariables : computer, password...

\footnotetext{
${ }^{1}$ Nous utilisons ici les termes marqué et non marqué dans un sens purement formel : marqué 'qui porte une marque' ; non marqué 'qui ne porte pas de marque'.

2 Pour être tout à fait complet il faut aussi mentionner les noms masculins ayant un pluriel féminin en $-a$ : l'uovo 'l'œuf', le uova 'les oeufs', ou ayant un double pluriel : il muro 'le mur', i muri 'les murs', le mura 'les remparts' (environ 25 lexèmes).
} 
En français enfin, la plupart des noms sont invariables, le pluriel est marqué uniquement par l'article ou par un autre déterminant antéposé au nom. Il existe également, en nombre relativement faible, des noms qui possèdent un pluriel par flexion interne : noms en -/al/ de

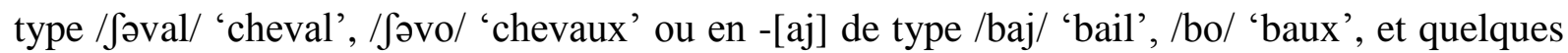
noms isolés : /œj/ 'œil', /jø/ 'yeux' ; /bœf/ 'bœuf' ; /bø/ 'bœufs' ; /os/ 'os', /o/ '(des) os'...

Dans certaines variétés romanes vernaculaires, on rencontre des systèmes plus complexes. L'occitan, si l'on considère l'ensemble de ses variétés, offre une grande diversité de configurations phonologiques et morphosyntaxiques. Ceci a pour conséquence que le marquage du nombre y présente une importante variation et, dans certains parlers nordoccitans, une grande complexité.

Trois types de marquage - mutuellement non exclusifs - sont possibles (cf. Sauzet 2011, Sibille 2011 et Barra-Jover 2012) :

(1) Par le seul déterminant antéposé, le substantif étant invariable. C'est le cas, par exemple en provençal : /la v'aks/3 'la vache', /lei v'aks/ 'les vaches'; / 'ome/ 'l'homme', /leiz 'ome/ ;

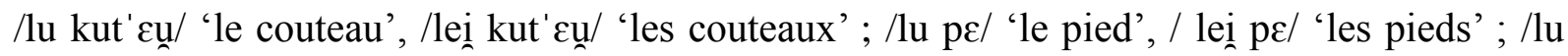
bras/ 'le bras', /lei bras/ 'les bras'.

(2) Par une marque /s/, agglutinée au substantif et redondante avec le déterminant. Cette marque peut apparaître sous la forme de différents allophones ([s], [z], [j], [h]...) ou allomorphes (-/s/, -/es/, -/ses/) : /la b'aks/ 'la vache', /laj b'akos/ 'les vaches'; /1 'ome/

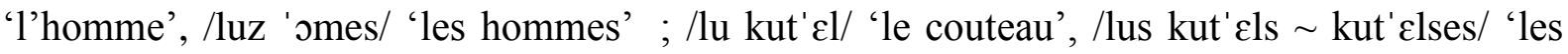
couteaux' ; /lu pe/ 'le pied', /lus pes/ 'les pieds' ; /lu bras/ 'le bras', /luj br' ases/ 'les bras'. Les parlers gascons et les parlers languedociens méridionaux sont représentatifs de ce type de marquage.

(3) Par des phénomènes de nature vocalique et/ou suprasegmentaux : allongement vocalique, alternance vocalique, déplacement d'accent, voire peut-être marque tonale ${ }^{4}$, le plus souvent redondants avec le déterminant ${ }^{5}$ : /lo v'atso/ 'la vache', /la: vots'a:/ 'les vaches'; /1 'ome/ 'l'homme' /1 'ome/, /lu: om'ej/ ; /lu kut' $\varepsilon /$ 'le couteau', /lu: kutj'au/ 'les couteaux'; /lu pe/ 'le pied', /lu: pe:/ 'les pieds'. Ce type de marquage se rencontre dans les dialectes nord occitans, en particulier en limousin.

On peut aussi distinguer trois types de systèmes :

(1) Des systèmes à marque « distribuée » (cf. Sauzet 2011) dans lesquels la marque du pluriel sur le nom ou l'adjectif est systématique.

(2) Des systèmes à marque non-distribuée dans lesquels la marque du pluriel sur le substantif ou l'adjectif est toujours absente.

\footnotetext{
${ }^{3}$ Pour des raisons à la fois pratiques et théoriques, et conformément à un usage qui tend à se répandre dans les travaux de dialectologie romane, nous préférons indiquer l'accent tonique devant la voyelle, plutôt que devant la syllabe accentuée.

4 L'hypothèse d'une marque tonale du pluriel dans certains parlers périgourdins est développée par Sauzet 2011.

${ }^{5}$ Dans certains cas rares et dans des contextes déterminés, le nom seul porte la marque du pluriel, comme c'est le cas dans le parler de Saint-Victor-en-Vivarais [la v'atso bl'anko] 'la vache blanche', [la v'atsa bl'anko] 'les vaches blanches' (Calvet 69, cité par Sauzet 2011) ou dans certains parlers limousins: [1 'ome] l'òme 'l'homme', [1 om'ej] l'òmes 'les hommes'.
} 
(3) Des systèmes «distribués filtrés » dans lesquels le pluriel est marqué sur certaines classes de noms ou adjectifs et pas sur d'autres.

La complexité de certains systèmes de marquage du nombre dans les dialectes nord-occitans peut être illustrée par les parlers de la Haute Vallée de Suse (Alta Valsusa en italien) et de la Haute Vallée du Cluson (Alta Val Chisone), en Italie. Ces deux vallées se situent à l'extrême nord-est du domaine linguistique occitan et confinent, au nord, avec l'aire linguistique du francoprovençal et à l'est, avec celle du piémontais, dialecte de type gallo-italique ${ }^{6}$. Jadis parties intégrantes du Dauphiné, elles ont été cédées par la France à l'État de Piémont-Savoie, lors du traité d'Utrecht en 1713 et sont aujourd'hui italiennes. La langue dominante (véhiculaire, administrative, scolaire) y est restée le français jusque dans les années 1880.

Nous décrirons dans un premier temps le système de marquage du nombre dans le parler des Ramats (commune de Chiomonte), puis la variation sur l'ensemble de la zone (Haute Vallée de Suse et Haut-Cluson) à travers une série d'exemples. Les données utilisées pour les Ramats sont issues d'enquêtes que nous avons effectuées en 2011 et 2012 et de documents sonores enregistrés en 1984.

La commune de Chiomonte (anciennement Chaumont) est la dernière commune occitanophone à la fois au nord et à l'est. À trois kilomètres du bourg de Chiomonte, en suivant la route nationale, le village de Gravere est de langue francoprovençale et à sept kilomètres, la ville de Suse constitue une enclave piémontaise en zone francoprovençale. La limite entre les communes de Chiomonte d'une part, et de Gravere et Giaglione d'autre part, suit l'ancienne frontière entre la France et l'État de Piémont-Savoie. Le village des Ramats fait partie de la commune de Chiomonte. Il est constitué de plusieurs hameaux, à l'écart dans la montagne, à quatre ou cinq kilomètres - suivant le hameau dans lequel on se rend - du bourg de Chiomonte. L'église paroissiale des Ramats est située à environ un kilomètre de la limite avec la commune de Giaglione, village de langue francoprovençale où l'on peut se rendre à pied en un peu plus d'une heure (il n'y a pas de route directe).

\section{Le marquage du nombre dans le parler des Ramats}

\subsection{Adjectifs postposés et substantifs}

\subsubsection{Noms et adjectifs invariables}

Sont invariables :

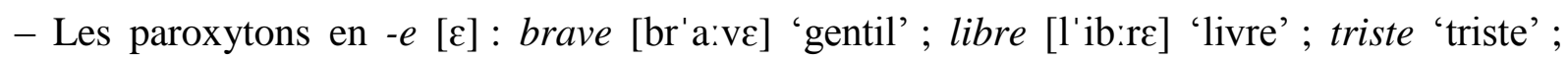
preire 'prêtre' ; sorre 'sœur' ; ainsi que les quelques rares paroxytons terminés par -ou $[\mathrm{u}]$ ou - $i$ [i] : counsou 'maire', chinevou 'chanvre', ari 'âne', soldi 'sou' (pour les conventions graphiques, voir annexe 1).

- Les paroxytons terminés par la voyelle nasale [ã] : óman ['omã] 'homme' ; joúvan 'jeune' (épicène) ; frèissan 'frêne' ; vórzan 'osier', fòussan 'faux' (adj). Il s'agit pour la plupart de mots dont les étymons se terminent par -INE, -ENE, -INU : OMINE, JUVENE, FRASSINU... et de quelques mots pour lesquels la voyelle nasale n'est pas explicable par l'étymologie comme

${ }^{6}$ Dialectes gallo-italiques : dialectes d'Italie, au nord d'une ligne La Spezia - Rimini, à l'exclusion du vénitien : piémontais, lombard, émilien, romagnol, ligurien. Certaines variétés de ligurien sont parlées en territoire français, dans la haute vallée de la Roya (Alpes-Maritimes) et à Bonifacio en Corse, ainsi qu'à Monaco. 
fóussan [f' gaulois *WORRIKE). Ce type ne concerne qu'un petit nombre de mots, il constitue une spécificité des parlers des Ramats et de Chiomonte, partagée avec les parlers francoprovençaux limitrophes (Giaglione: ['omen] 'homme'; [d'ovey] 'jeune'; Vayr/Telmon 2004), mais qui n'est présent dans aucun autre parler occitan de la zone.

- Les paroxytons en ['VjV] : úiou ['y:ju] 'œuf; : búiou 'bœuf' ; al miou [al m'i.' ${ }^{\mathrm{j}} \mathrm{u}$ 'le mien', oiou ['ø:ju] 'huile; foudie [fud'i.'. ${ }^{\mathrm{j}} \varepsilon$ ] 'tablier'; fie 'fil'; clouchie 'clocher' ; charbounie 'charbonnier'. Ces paroxytons proviennent, pour la plupart, de la dissociation des composants d'une diphtongue ou d'une triphtongue ou, dans le cas de xiou, de la chute de [1] devant [j] : OLEU $>[$ 'olju $]>[$ ' $\varnothing \mathrm{ju}]$. La dissociation des composants de certaines diphtongues ou triphongues est propre aux parlers de Chiomonte et des Ramats, les autres parlers occitans de la zone les conservent intactes : [byun], [miun], [fudj'e(1)].

- Les oxytons terminés par une voyelle nasale : pan [pã] 'pain' (<PANE) ; mourin 'moulin' (<MOLINU) ; meisoun 'maison' (< MANTIONE), poun 'pomme' (< POMU) ; chan 'champ' (< CAMPU), ploun 'plomb' (< PLUMBU $)^{7}$.

- Les oxytons terminés par une voyelle longue ou une diphtongue : jô̂ 'jour' [zu:], bô 'bois' [bo:], pô 'planche', jalôu 'jaloux', dourô̂ 'douleur, nâa 'nez', sâ 'sel', chinâ 'rigole', châa 'cher', mouvê 'mauvais', partû 'trou', linsô 'drap', clòu [klow] 'clé', piòu 'poil', frei frê 'frais'. Les types étymologiques ayant abouti à des finales longues sont ceux dont la voyelle tonique est suivie de S ou R (intervocaliques ou suivis d'une autre consonne) ou de L simple intervocalique : DIURNU > jorn > jổ 'jour'; BOSK > bosc > bô 'bois'; POSTE > post > pô 'planche'; DOLORE > dołor > dourô̂ 'douleur'; NASU > nas >nâ 'nez'; SALE > sat > $s \hat{a}$ 'sel'; CANALE > chanat > chinâ 'rigole'; CARU > char > châ 'cher'; déverbal de PERTUSIARE > pertus $>$ part $\hat{u}$ 'trou' ; LINTOLEU > lençot > linsô 'drap' (jaloû est un emprunt au français, la forme attendu dans le dialecte serait *jiroû [ziru:] < *ZELOSU). Le mot 'fusil' - qui a au singulier la forme fusil là où l'on attendrait fusî - fait au pluriel fusî. Il convient de préciser que les oppositions de longueur vocalique qui sont/étaient très nettement perceptibles chez les plus vieilles générations, le sont moins chez les générations plus jeunes, en particulier celles nées après la seconde guerre mondiale, qui ne les réalisent que sporadiquement de façon audible ; toutefois, l'opposition $-a /-\hat{a}$ reste toujours perceptible car elle se double d'une opposition de timbre , $-a$ tonique bref $^{8}$ final est postérieur : ['a], tandis que $-\hat{a}$ tonique long est plus central : ['a:]. De même, l'allongement de [' $\varepsilon]$ tonique final donne ['e:] : pè [pe] 'pied', pê [pe:] 'pieds'.

- Les oxytons terminés par une consonne autre que $[\mathrm{t}],[1],[K],[\mathrm{s}]$ ou par un groupe de consonnes : sap [sap] 'sapin'; toc [tok] 'morceau'; fioc [fjok] 'feu' ; cuver [kuv'er] 'toit' ; pourast [pur' ast] 'poulet'; ours [urs] 'ours'.

\subsubsection{Noms et adjectifs fléchis en nombre}

${ }^{7}$ Les données de Hirsch 1978 pour le bourg de Chiomonte (qui datent du début des années 1960) et certains témoignages, semblent montrer qu'il a pu exister des nasales longues au pluriel : [pã] 'pain', [pã:] 'pains' (noté

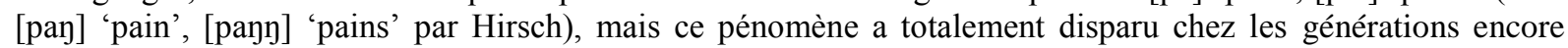
vivantes.

${ }^{8}$ L'opposition [a] - [a:] existe également en ce qui concerne l'article défini féminin : sing. [la], pl. [la:], bien qu'il ne soit pas accentué. 
Les noms et adjectifs fléchis en nombre se répartissent en plusieurs catégories :

- Les paroxytons féminins en -o $(<\mathrm{A})$ font leur pluriel en - $e$ : eitjero [eitf' e: ro] 'étoile', eitjere [eiff'e:re] 'étoiles'; bravo 'gentille', brave 'gentilles'... Le substantif momo et sa variante moma 'mère, maman' font au pluriel mome, tandis de popo popa 'père, papa', qui est masculin, reste invariable.

- Les oxytons terminés par une voyelle brève font leur pluriel par allongement vocalique : pra [pra] 'pré', prâ [pra:] 'prés ; pè [pe] 'pied', pê [pe:] 'pieds'; chourí [Jur'i] 'chevreau', chourî [Jur'i:] 'chevreaux', patanú [patan'y] 'nu', patanû [patan'y:] 'nus'. Font partie de cette catégorie les participes passés réguliers : parlá 'parlé, parlée', parlâ 'parlés, parlées' ; vandú 'vendu', vandî 'vendus'; sourtí 'sorti', sourtî 'sortis'. Ces noms ou adjectifs sont issus d'étymons présentant un $\mathrm{T}$ ou un $\mathrm{D}$ simple intervocalique après la voyelle tonique : PRATU, PEDE, NUDU, participes en -ATU, -UTU, -ITU... Rappelons qu'en occitan vivaro-alpin le traitement des occlusives sourdes intervocaliques ou intervocaliques devenues finales (finales romanes) du latin diffère, d'une part, des autres dialectes occitans et d'autre part, du français et du francoprovençal (Sibille 2004). En français et en francoprovençal il y a lénition complète de $\mathrm{T}$ et $\mathrm{C}[\mathrm{k}]$, tandis que $\mathrm{P}$ aboutit à $[\mathrm{v}]$ en position intervocalique, et s'amuït en finale romane, comme dans $\operatorname{lou}(p)^{9}$ (< LUPU), ou aboutit à [f], comme dans chef (< CAPUT). Dans les dialectes occitans autres que le vivaro-alpin, ces consonnes se sont sonorisées à l'intervocalique, et sont redevenues sourdes en finale romane (il n'existe pas, en occitan, d'occlusive sonore en position de coda finale) : PRATU > pradu > prad > prat. En vivaro-alpin on a un traitement mixte : à l'intervocalique $\mathrm{P}$ se sonorise tandis qu'il y a chute de $\mathrm{T}$ et $\mathrm{C}[\mathrm{k}]$; en finale romane $\mathrm{P}$ et $\mathrm{C}[\mathrm{k}]$ se maintiennent, tandis qu'il y a chute de $\mathrm{T}$ :

\begin{tabular}{|c|c|c|c|c|c|c|}
\hline & $\begin{array}{l}\text { LUPU, } \\
\text { 'loup' }\end{array}$ & $\begin{array}{c}\text { LUPA } \\
\text { 'louve' }\end{array}$ & $\begin{array}{l}\text { AMATU } \\
\text { 'aimé' }\end{array}$ & $\begin{array}{l}\text { AMATA } \\
\text { 'aimée' }\end{array}$ & $\begin{array}{l}\text { JOCU } \\
\text { 'jeu' }\end{array}$ & $\begin{array}{c}\text { *JOCARE } \\
\text { 'jouer' }\end{array}$ \\
\hline oc. référentiel & [lup] & [l'ubo] & [aim'at] & [aim'ada] & [djok] & [dzug'a] \\
\hline vivaro-alpin & [lup] & [1'ubo] & [aim'a] & [aim'aa] & [dुok] & [dzu'a] \\
\hline français & {$[\mathrm{lu}]$ ou $[1 \varnothing]$} & [l'uvə] & [عm'e] & {$\left[\varepsilon \mathrm{m}^{\prime} \mathrm{e}(\partial)\right]$} & {$[3 \varnothing]$} & [zu'e] \\
\hline
\end{tabular}

Le traitement différent de $\mathrm{T}$ d'une part et de $\mathrm{P}$ et $\mathrm{K}$ d'autre part, en finale romane, explique que, dans le parler des Ramats, les substantifs issus d'un étymon en - 'VtV et ceux en ' $\mathrm{VpV}$ ou ' $\mathrm{VkV}$ aboutissent à deux types morphologiques différents, le premier formant le pluriel par allongement vocalique, le second comportant des lexèmes invariables au pluriel :

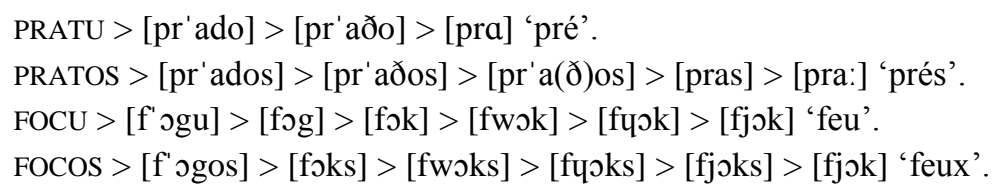

- Les oxytons terminés par -al [al] (<-ALLU, -ALLE), ò [ol] (<-OLLU, -OLLE), -alh [aK], font leur pluriel en òu : chaval 'cheval', chavòu 'chevaux'; fol 'fou', fòu 'fous'; mol 'mou', mòu 'mous'; travalh [trav'aא] 'travail', travòu 'travaux'; jalh 'coq', jòu 'coqs'. Le mot dalh '(la) faux', toutefois, fait au pluriel diòu, au lieu de la forme attendue : *dòu. Les emprunts savants au latin ou les emprunts à des langues modernes, sont généralement intégrés au système : capoural 'caporal', capouròu 'caporaux'.

\footnotetext{
${ }^{9}$ En français, le $p$ de loup est savant et purement orthographique, en ancien français on a lou ou leu.
} 
- Les oxytons issus d'un étymon en -ELLU, -ELLE, ILLU font leur pluriel -eiou ['e:ju]. Dans le bourg de Chiomonte la finale [ $[\mathrm{l}]$ reste bien distincte de [al], aux Ramats elle a tendance à passer à [al], mais ce n'est pas systématique chez tous les locuteurs: chapal chapel 'chapeau', chapeiou 'chapeaux' ${ }^{10}$; bal bel 'beau', beiou 'beaux'; apal apel 'peau', apeiou 'peaux'. Les emprunts sont généralement intégrés au système : otel 'hôtel', oteiou 'hôtels'.

- Les oxytons terminés par -elh $[\varepsilon \kappa]$ ou -elh $[\varnothing K]$ ont un pluriel en -iòu : velh [veK] 'vieux' (sing.) (< VET(U)LU), viòu [vjow] 'vieux' (pl.) ; felh 'feuille (de papier)', fiòu 'feuilles' ;

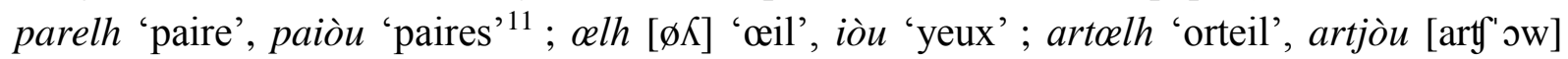
'orteils' ${ }^{12}$. Toutefois sourelh 'soleil' fait - semble-t-il - au pluriel soureiou [sur'e:ju] 'soleils', là ou l'on attendrait *souiòu. Nous avons trouvé un seul exemple de mot en -ilh [iK] : ambirilh 'nombril', qui, selon nos informateurs, est invariable ${ }^{13}$.

- Les substantifs, adjectifs et participes féminins en -ió [j'o], djó [d马'o], tjó [t' en $-i e$ [ $i{ }^{j} \mathrm{j} \varepsilon$ ]. Il s'agit de mots originellement paroxytons, devenus oxytons par déplacement de l'accent tonique sur la finale : maratio [marat ${ }^{1}{ }^{j}$ o] 'maladie' > maratjó [maratf' le schéma paroxytonique est conservé : mindjó [mindz'o] 'fille', mindie [mind'i.j ${ }^{j}$ ] 'filles'; maratjó 'maladie', maratie 'maladies'; patanió [patanj' 'o] 'nue', patanuie [patan'y:je] 'nues'; vandjó 'vendue', vanduie 'vendues'; andourmió 'endormie', andourmie 'endormies'. Avec le suffixe -iió [ij' 'o] '-erie', le phonème $r / \mathrm{r} /$, amuï devant [j], réapparait au pluriel : boulanjiió [bulãzij'o] 'boulangerie', boulanjirie [bulãzir'i ${ }^{j}{ }^{j} \varepsilon$ ] 'boulangeries'; pourquiió 'porcherie', pourquirie 'porcheries'. Pour cette catégorie de lexèmes, la forme pleine de la base est celle du pluriel (et du masculin en ce qui concerne les participes), la base du féminin singulier étant une forme contracte qui ne permet pas de prévoir la forme exacte de la base du pluriel, alors que cette dernière permet de prévoir celle du singulier.

- Les substantifs et adjectifs oxytons en - 'Vt ont un pluriel en -'Vs : bot [bot] 'garçon, fils', bos [bos] 'garçons, fils' ; surat suret (SOLU + ITTU), soures 'seuls' ; chat [Jat] 'chat' (< CATTU), chas [Jas] 'chats'; muet [my'et] 'muet' (emprunt au français), mues [my'es] 'muets' ; gout 'goût' (< GUSTU), gous 'goûts' ; let 'lit' (< LECTU), les 'lits' ; fret 'froid' (< FRIG(I)DU), fres 'froids'; dret 'droit' (< DIRECTU), dres 'droits'; lèit [leit] 'lait' (< LACTE), lèis [leins] 'laits'; noet 'nuit' (< NOCTE), noes 'nuits'. D'un point de vue diachronique, il y a eu effacement de la consonne finale de la base devant/s/ marque du pluriel, mais d'un point de vue strictement synchronique, on doit considérer qu'on est en présence d'un phénomène de flexion interne : lorsqu'une base se termine par - 'Vt au singulier, /t/ alterne avec /s/ au pluriel. En effet, [s] n'alterne qu'avec [t] (sap, fioc... sont invariables) et aucun pluriel ne s'obtient par agglutination d'une marque /s/ à une base, on doit donc considérer que [t] alterne avec [s] tout comme [al] alterne avec [oun] dans chaval / chavòu, ou [El] avec ['e:ju] dans chapel / chapeiou... Par exception, tout 'tout' fait touti au masculin pluriel.

\subsubsection{Cas particulier des noms terminés par [s]}

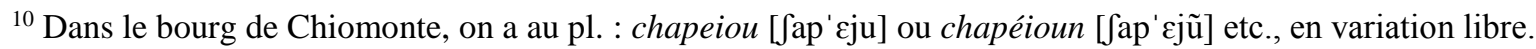

11 [ $]$ s'amuït régulièrement devant $[\mathrm{j}]$.

$12[\mathrm{t}]+[\mathrm{j}]$ aboutit régulièrement à $[\mathrm{t}]$ et $[\mathrm{d}]+[\mathrm{j}]$ à $[\mathrm{d}]$.

13 Pour ces deux mots : sourelh 'soleil' et ambirilh 'nombril', nos informateurs manifestent une certaine hésitation : il n'est pas très naturel de parler de soleils ou de nombrils au pluriel.
} 
En ce qui concerne le pluriel des oxytons terminés par [s] au singulier, on constate une certaine irrégularité et parfois une hésitation de la part des locuteurs : courbas 'corbeau' (< CORVU + ACCIU), òumas (òman + ACCIU) it. 'omaccio', armas 'balais' (déverbal de armassâ 'ramasser') sont invariables ; bachas 'vasque (de la fontaine)' fait au pluriel bachòu ou bachas; boutjas ( bot + ACCIU) it. 'ragazzone' fait boutjòu; tandis que bras 'bras' (< BRACCIU) et purus 'poire' font respectivement brâ et purû.

\subsubsection{Synthèse}

Le parler des Ramats présente un système de marquage du nombre à marque distribuéefiltrée. Pour les substantifs et les adjectifs qualificatifs variables en nombre, plusieurs procédés de marquage du pluriel sont mis en œuvre :

- Marquage par allongement vocalique : pra [pra] 'pré', prâ [pra:] 'prés'.

- Marquage par un suffixe vocalique alternant : -ole : vacho 'vache', vache 'vaches'

- Marquage par flexion interne (modification de la base) : chaval 'cheval', chavòu 'chevaux' ; bot 'garçon', bos 'garçons'...

- Marquage combinant suffixe alternant et déplacement de l'accent tonique : andourmió [ãdurmj' ] 'endormie', andourmie [ãdurm' i: ${ }^{\mathrm{j}} \varepsilon$ ] 'endormies'.

Les substantifs et adjectifs qualificatifs (ou participes) variables en nombre peuvent être répartis en neuf classes :

(1) Substantifs et adjectifs féminins paroxytons en -o au singulier: vacho 'vache', vache 'vaches'.

(2) Substantifs et adjectifs oxytons terminés par une voyelle brève au singulier : pra 'pré', prâ 'prés'.

(3) Substantifs et adjectifs oxytons terminés par -al, òl, -alh au singulier : chaval 'cheval', chavòu 'chevaux' ; fol 'fou', fòu 'fous'; travalh 'travail', travòu 'travaux' .

(4) Substantifs et adjectifs oxytons terminés par -al -el au singulier: chapal chapel 'chapeau', chapeiou 'chapeaux.

(5) Substantifs et adjectifs oxytons terminés par -elh ou -oelh : velh 'vieux' (s.), viòu 'vieux' (pl.) ; celh 'oeil', iòu 'yeux'.

(6) Substantifs et adjectifs oxytons terminés par une voyelle suivie de [t] : bot 'garçon', bos 'garçons'.

(7) Substantifs, adjectifs et participes féminins terminés par -ió [j'o], -tjó [t' o] ou -djó [d马'o], au singulier : maratjó 'maladie', maratie 'maladies'

(8) Substantifs terminés par $-s$ [s] au singulier avec effacement de $-s$ et allongement vocalique au pluriel : bras 'bras' (s.), brâ 'bras' (pl.).

(9) Substantifs terminés par $-s[\mathrm{~s}]$ au singulier, faisant leur pluriel en -òu: boutjas 'ragazzone', boutjòu 'ragazzoni'.

Les classe 8 et 9 ne regroupent pas tous les substantifs terminés par $-s$ au singulier, certains sont invariables comme courbas 'corbeau'.

On note, en outre, quelques rares irrégularités, par exemple boulet 'champignon' fait au pluriel bouiòu là où l'on attendrait *boules; dalh '(la) faux' fait au pluriel diòu là où on attendrait *dòu...

Les substantifs et adjectifs qualificatifs autres que ceux appartenant aux neuf classes énumérées ci-dessus (désormais 'classe 10'), sont invariables; il s'agit : 
- des paroxytons autres que ceux appartenant à la classe 1,

- des oxytons se terminant par une voyelle nasale, une voyelle longue, une diphtongue, une consonne autre que $l[1], l h[K], t[\mathrm{t}], s[\mathrm{~s}]$ ou par un groupe de consonnes.

- des oxytons terminés par [s] $-s$ autre que ceux appartenant aux classes 8 et 9 .

L'appartenance d'un substantif terminé par $-s$ [s] au singulier aux classes 8, 9 ou 10 n'est prévisible ni à partir du singulier, ni à partir du pluriel.

\subsection{Déterminants et adjectifs antéposés.}

\subsubsection{Adjectifs et déterminants oxytons}

En phonétique syntactique, devant une consonne, la consonne finale des adjectifs et déterminants antéposés au nom, s'efface : oul ei bichit 'il est petit', $i$ soun bichis 'ils sont petits', mais : in bichí bot 'un petit garçon', dô̂ bichí bos 'deux petits garçons'. Si la consonne finale de l'adjectif ou du déterminant est [1], la voyelle s'allonge : oul ei bal bel 'il est beau', mais in bê bot 'un beau garçon'; quel óman 'cet homme', mais quê bot 'ce garçon'.

\subsubsection{Adjectifs et déterminants paroxytons}

En phonétique syntactique, $-o[0]$ et $-e[\varepsilon]$ post toniques, tendent à s'effacer après un accent secondaire (le plus souvent ils s'amuïssent complètement, parfois il subsiste un [ə] faiblement audible) : ex. ou parlo [u p'arlo] 'il parle', mais : ou parlë pâ [u p, $\operatorname{arl}^{(ə)} \mathrm{p}$ 'a:] 'il ne parle pas'. En ce qui concerne les adjectifs et déterminants antéposés, le phénomène se produit au singulier mais pas au pluriel :

oul ei pòure [ul ei p' ource] 'il est pauvre', mais in pòurë bot [ĩ pour bot] 'un pauvre garçon'. oul ei brave [ul ei br'a:ve] 'il est gentil' in brau bot [ĩ brau bot] 'un gentil garçon'

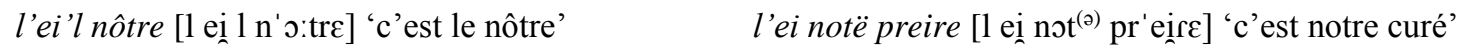

il ei pòuro [il ei p' ouro] 'elle est pauvre' inë pòurë mindjó [in pour mindz'o] 'une pauvre fille'

il ei balo [il ei b'al:o] 'elle est belle'

inë balë mindjó [in bal minds' o] 'une belle fille'

En revanche, au pluriel, on aura :

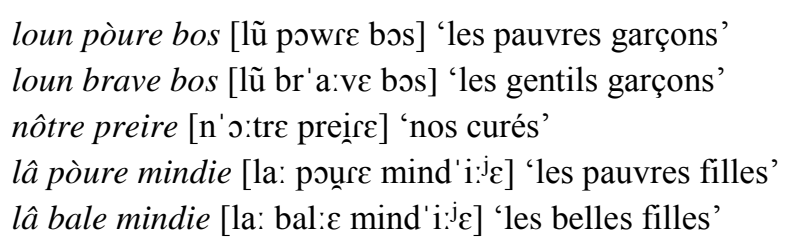

Dans certains cas - comme on peut le remarquer dans les exemples ci-dessus -, la lénition de la voyelle finale du singulier provoque une modification de la base : brave [br'ave] devient brau [brau], nôtre [n'o:tre] devient notë [not $\left.{ }^{(\vartheta)}\right]$.

Devant un nom commençant par une voyelle, la voyelle finale de l'adjectif est élidée au singulier : in pòur' eifan 'un pauvre enfant', inë bal' assieto 'une belle assiette' ; tandis qu'au pluriel il y a résurgence de $-s[\mathrm{z}]$, ancienne marque du pluriel : loun pòures eifan [lũ $\mathrm{p}_{\mathrm{o}} \mathrm{owr \varepsilon z}$ eif' ã] 'les pauvres enfants'; lâ bales assiete [la: b, allez assi 'et: $\varepsilon$ ] 'les belles assiettes'.

On voit donc qu'en ce qui concerne les adjectifs intrinsèquement paroxytons (c'est-à-dire paroxytons non seulement au féminin, mais aussi au masculin, comme pòure, brave), la distinction masculin singulier / féminin singulier est neutralisée lorsqu'ils sont antéposés. En revanche, dans cette position, les formes du masculin singulier et du pluriel sont différenciées, alors qu'elles ne le sont pas lorsqu'ils sont postposés : 


Postposé
\begin{tabular}{|c|c|c|}
\hline & $\mathrm{M}$ & $\mathrm{F}$ \\
\hline $\mathrm{S}$ & \multirow{2}{*}{ pòure } & pòuro \\
\hline $\mathrm{P}$ & pòre \\
\end{tabular}

Antéposé $+\mathrm{C}$
\begin{tabular}{|l|c|c|}
\hline & $\mathrm{M}$ & $\mathrm{F}$ \\
\hline $\mathrm{S}$ & \multicolumn{2}{|c|}{ pòurë } \\
\hline $\mathrm{P}$ & \multicolumn{2}{|c|}{ pòure } \\
\hline
\end{tabular}

Antéposé $+\mathrm{V}$
\begin{tabular}{|l|c|c|}
\hline & $\mathrm{M}$ & $\mathrm{F}$ \\
\hline $\mathrm{S}$ & \multicolumn{2}{|c|}{ pòur' } \\
\hline $\mathrm{P}$ & \multicolumn{2}{|c|}{ pòures } \\
\hline
\end{tabular}

Cette distribution différente des marques constitue une originalité du parler des Ramats et de Chiomonte par rapport aux autres parlers occitans de la Haute Vallée de Suse et de la vallée voisine du Haut Cluson, dans lesquels il n'y a pas de variation des formes ni de leur distribution en fonction de la position :

Salbertrand
\begin{tabular}{|l|c|c|}
\hline & M & F \\
\hline S & paurë & paurä \\
\hline $\mathrm{P}$ & pauri & paura \\
\hline
\end{tabular}

Champlas-du-Col ${ }^{14}$

Oulx
\begin{tabular}{|l|c|c|}
\hline & $\mathrm{M}$ & $\mathrm{F}$ \\
\hline $\mathrm{S}$ & \multicolumn{2}{|c|}{ paurë } \\
\hline $\mathrm{P}$ & paure & paura \\
\hline
\end{tabular}

Pragelà
\begin{tabular}{|l|c|c|}
\hline & $\mathrm{M}$ & $\mathrm{F}$ \\
\hline $\mathrm{S}$ & \multicolumn{2}{|c|}{ paurë } \\
\hline $\mathrm{P}$ & pauri & paura \\
\hline
\end{tabular}

Le parler des Ramats et de Chiomonte est également le seul qui ait une forme unique pour le pluriel. La convergence des formes du masculin pluriel et du féminin pluriel s'explique par une tendance à l'ouverture de -e final post tonique qui présente un spectre de réalisation assez large : $[\varepsilon] . . .[3] \ldots[\mathfrak{x}]$, qui a pu recouvrir partiellement celui de $-a$ final post tonique : [æ]...[a], tendant ainsi à neutraliser l'opposition entre les deux phonèmes.

\subsection{Cas particuliers.}

Quelques lexèmes grammaticaux et quelques adjectifs indéfinis variables en genre et en nombre ont une flexion particulière :

- L'article défini présente une marque -oun [ũ] au masculin pluriel et -âa $[\mathrm{a}:]$ au féminin pluriel

$$
\begin{aligned}
& \text { (a) } l[(\mathrm{a}) 1]^{15}+\mathrm{C} ; l \text { ' }[1]+\mathrm{V}: \text { 'le'. } \\
& \text { loun }[\mathrm{lu}]+\mathrm{C} ; \text { louns }[1 \mathrm{u} z]+\mathrm{V}: \text { 'les' (masc.) } \\
& \text { la }[\mathrm{la}]+\mathrm{C} ; l \text { ' }[1]+\mathrm{V}: \text { 'la'. } \\
& \text { lâ [la:] + C ; las }[\mathrm{laz}]+\mathrm{V} \text { : 'les' (fém.) }
\end{aligned}
$$

- Le démonstratif présente, comme l'article défini, une marque -oun(s) au masculin pluriel, ainsi qu'une base spécifique au féminin singulier. La base de l'adjectif démonstratif possède un forme contracte au pluriel, en variation libre avec la forme pleine :

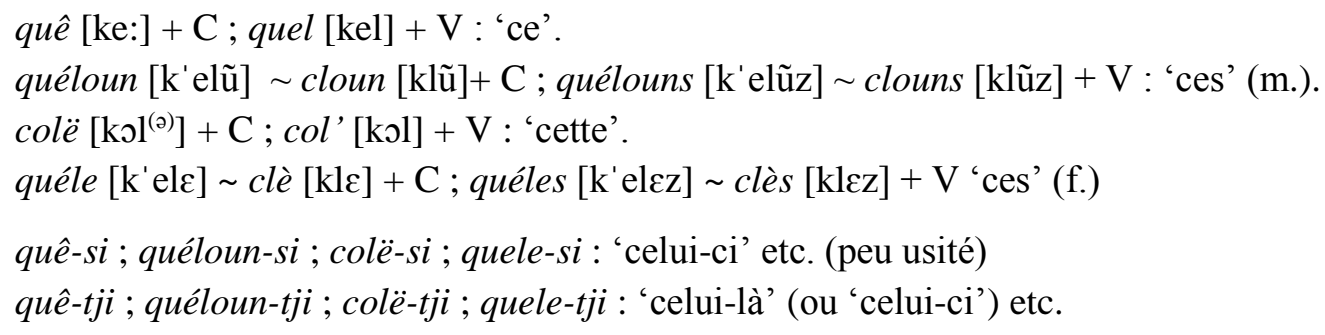

${ }^{14}$ Commune de Sestrières.

${ }^{15} \mathrm{Al}$ [al] après une pause ou une consonne, ' $l$ après voyelle. 
quê-lâ ; quéloun-lâ ; colë-lâ ; quele-lâ : 'celui-là, là bas' etc.

- Le pronom accentué de troisième personne présente également une marque -oun au masculin pluriel, ainsi qu'une marque $-i$ au féminin singulier.

iê 'lui' ; iáloun 'eux' ; iali 'elle'16 ; iale 'elles'.

- Ont une marque $-i(s)[\mathrm{i}(\mathrm{z})]$ au masculin pluriel :

tout 'tout'; touti 'tous' ; touto 'toute'; toute 'toutes'.

òutre 'autre' (m.) ; òutri 'autres' (m.), òutro 'autre' (f.), òutre 'autres' (f.).

can, canti, canto, cante 'combien'.

tan, tanti, tanto, tante 'tant' 17

\section{Variation des systèmes de marquage du pluriel dans les hautes vallées du Suse et du Cluson.}

Nous proposons d'analyser la variation des systèmes de marquage du pluriel dans les hautes vallées du Suse et du Cluson, à travers les exemples figurant dans les tableaux ci-dessous ${ }^{18}$, qui renvoient à différents types d'étymons, dans les parlers des localités suivantes: Fénestrelle, Pragelà (Pragelato en italien), Champlas-du-Col (commune de Sestrières), Les Ramats (Commune de Chiomonte), Salbertrand, Oulx, Bardonnèche, Rochemolles. Lorsque deux formes sont citées, la première est celle du singulier, la seconde celle du pluriel; lorsqu'une seule forme est citée, le substantif est invariable. Les données utilisées sont issues de : Baccon-Bouvet 1989 (Salbertrand), Talmon 1914 et Berton et al. 2003 (Pragelà), Bourlot et al. 2007 (Fénestrelle et Mentoules), Vignetta 1981 (Fénestrelle), Castagno et al. 2003 (Champlas), Gleise-Bellet 2003 (Bardonnèche), Hirsch 1978 (pour l'ensemble des localités), Jayme 2003 et Perron 1984 (Oulx), Masset 1997 (Rochemolles).

\begin{tabular}{|c|c|c|c|c|c|c|}
\hline & 'pain' & 'gras' & 'jour' & 'pré' & 'seigle' & 'bois' \\
\hline étymon & PANE & CRASSU & DIURNU & PRATU & $*_{\text {BLAD }}$ & $*_{\mathrm{BOSK}}$ \\
\hline Fénestrelle & pan, pans & grô & djourn, djours & pra,prô & bla, blô & bóoc, bóocs \\
\hline Pragelà & pon & grô & dzourn, dzours & pra, prô & bla, blô & bóoc, bóocs \\
\hline Champlas & pån, påns & gra & djourt, djours & prå, pra & blå, bla & bóoc, bóocs \\
\hline Ramats & pan & grâ & jôे & pra, prâ & bla, blâ & $b \hat{o}$ \\
\hline Salbertrand & pan & $g r a \hat{a}$ & jôे & pra, prâ & bla, blâ & $b \hat{o}$ \\
\hline Oulx & pan & grâ & jôे & pra,prâ & bla, blâ & $b \hat{o}$ \\
\hline Bardonnèche & pan & grâ & djôे & pra, prâ & - & $b \hat{o}$ \\
\hline Rochemolles & pan & grâ & jôे & pra, prâ & bla, blau & $b \hat{o}$ \\
\hline
\end{tabular}

${ }^{16}$ Le $-i$ de $i a l i$, est une ancienne marque du cas sujet féminin singulier de certains pronoms et déterminants, que l'on trouve dans des textes médiévaux de l'est occitan et de l'aire francoprovençale ; voir Martin 1974 et Sibille 2003, § 13.3.

${ }^{17}$ Tan et can peuvent s'accorder comme en italien ou se construire avec la préposition 'de' comme en français : tantes oure ou tan d'oure 'tant d'heures', canti bos ou can'd bos 'combien de garçons'.

${ }^{18}$ Ces tableaux concernent uniquement les oxytons ; pour les paroxytons, se reporter aux tableaux présentés plus haut, à la fin du $\S 2.2 .2$. 


\begin{tabular}{|c|c|c|c|c|c|}
\hline & 'chat' & 'paquet' & ‘sapin’ & 'toit' & 'dent' \\
\hline étymon & CATTU & pack + et & $*_{\text {SAPPU }}$ & COPERTU & DENTE \\
\hline Fénestrelle & tchôt, tchôs & paquet, paqués & sôp, sôp $(s)$ & cubert, cubers & dent, dens \\
\hline Pragelà & $t s \hat{o} t, t s \hat{o}$ & paquet, paqués & sôp, sôps & cubert, cubers & dent, den \\
\hline Champlas & tchèt, tchès & paquet, paqués & sèp, sèps & cubert, cubers & dënt, dëns \\
\hline Ramats & chat, chas & paquet, paqués & sap & cuver & dan \\
\hline Salbertrand & chat, chas & paqué, paqués & sap, sappi & cubê & dën \\
\hline Oulx & chattë, chas & - & sap, sappe & - & - \\
\hline Bardonnèche & tcha, tchase & paqué, paquiau & sap, sappe & cubé, cubiau & dan \\
\hline Rochemolles & chat, chas & paqué, paqués & sap & tjubé, tjubeu & dèn, den \\
\hline
\end{tabular}

\begin{tabular}{|c|c|c|c|c|c|}
\hline & 'troupeau' & 'cheval' & 'fou' & 'vieux' & 'drap' \\
\hline étymon & TROPPU+ELLU & CABALLU & FOLLE & VETULU & LINTOLEU \\
\hline Fénestrelle & troup-el, -eaus & cavòl, cavaus & fól, faus & velh, veus & - \\
\hline Pragelà & troup-el, -au & cavòl, cavau & fól, fau & velh, vei & linsol, linsols \\
\hline Champlas & troup-el,-iòus & tchaval, tchavaus & fol, fòus & - & - \\
\hline Ramats & troup-al, -eiou & chaval, chavòu & fol, fòu & velh, viòu & lins $\hat{o}$ \\
\hline Salbertrand & troup-è, -iòu & chavá, chavòu & fol, fòu & velh, viòu & linsô \\
\hline Oulx & troup-é, -iau & chavá, chavau & fol, fole & velh, velhe & linsô \\
\hline Bardonnèche & troup-é, -iòu & tchavá, tchavau & fol, fole & veu & - \\
\hline Rochemolles & troup-é, -éu & chavá, chavau & fól, fòu & volh, viòu & Ihinsóo, lhinsòu \\
\hline
\end{tabular}

Fénestrelle et Pragelà se situent dans la Haute Vallée du Cluson, Champlas-du-Col près du col de Sestrières (Sestriere en italien), qui permet la communication entre les deux vallées ; Les Ramats, Salbertrand et Oulx, dans la Haute Vallée de Suse ; Bardonnèche et Rochemolles dans une vallée latérale débouchant, à Oulx, dans la Haute Vallée de Suse (voir carte et tableau des distances en annexe).

\subsection{Haut Cluson et environs de Sestrières}

Les parlers du Haut Cluson et celui de Champlas-du-Col, phonétiquement plus conservateurs, ont conservé une marque $-s$ agglutinée au substantif pour certaines catégories de lexèmes : oxytons terminés par une voyelle nasale (ou post nasalisée suivant les parlers), pan [pa $\left.{ }^{\mathrm{p}}\right]$ 'pain', pans [pa $\left.{ }^{\natural} \mathrm{s}\right]$ 'pains'(à Fénestrelle et à Champlas, mais pas à Pragelà); par une consonne autre que [t], [1], [K], [s] : sôp [so:p] 'sapin', sôps [so:ps] 'sapins', booc [bo:k] 'bois' (s.), boocs [bo:ks] 'bois' (pl.) ; par un groupe de deux consonnes : cubert [ky'bert] 'toit', cubers [kybers] 'toits', djourn [ḑurn] 'jour', djours [ḑurs] 'jours'. Dans ce dernier cas, la consonne finale de la base s'efface au pluriel, conformément à un processus attesté dès l'époque médiévale sur l'ensemble de l'aire linguistique occitane. La consonne finale s'efface également dans les bases en - 'Vt ( $-s$ étant dans ces parlers, un morphème de pluriel isolable, agglutinée à certaines catégories de bases, on ne peut plus y analyser l'alternance $[\mathrm{t}]$ - $[\mathrm{s}]$ en termes de flexion interne comme nous l'avons fait pour le parler des Ramats).

À Fénestrelle et à Champlas, la marque [s] est également conservée au pluriel des noms et adjectifs en /el/, /al/, /ol/ (<-ELL-, -ALL-, -OLL-) : [trup'cl] 'troupeau', [trup'eaus] (Fénestrelle) - [trupj' 'ous] (Champlas) 'troupeaux' etc.

En revanche, dans les lexèmes terminés anciennement par une voyelle tonique suivie de /s/, il y a, comme dans les parlers de la Haute Vallées de Suse, chute de /s/ et allongement compensatoire (et dans une deuxième étape il y a passage de [a: à [๑:] à Pragela et Fénestrelle 
tandis qu'à Champlas la longueur n'est plus sensible mais il subsiste une opposition de timbre : /a/-/a/) : pra [pra] < [pra] 'pré', prô [pro:] < [pras] 'prés', mais grô [gro:] < [gras] 'gras' invariable (Pragelà et Fénestrelle) ; prå [pra] 'pré', pra [pra] 'prés', mais gra [gra] 'gras' invariable (Champlas).

À Pragelà, dans les lexèmes issus d'un étymon comportant un L simple (type LINTOLEU, PILU) ayant abouti à /1/ (L vélaire), /1/ est maintenu (ou passe à [e] chez les plus jeunes générations); au pluriel on a un /l/ «clair » suivi de /s/ selon les données de Talmon 1914 :

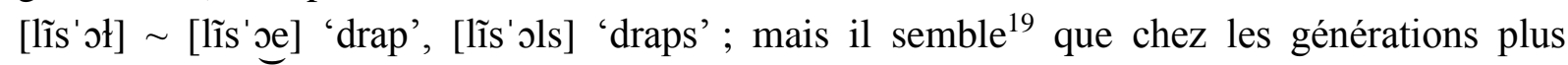
jeunes on trouve la forme [lĩs'

\subsection{Haute vallée de Suse.}

Par rapport aux parlers du Haut Cluson et des environs de Sestrières, le parler des Ramats présente un certain nombre d'évolutions que l'on retrouve dans les autres parlers de la Haute Vallée de Suse, cependant il apparaît plus conservateur que la plupart des autres parlers de cette vallée. En effet, les consonnes finales s'y maintiennent bien et il refuse un certain nombre d'innovations.

À Salbertrand, Oulx, Bardonnèche et Rochemolles, il y a chute de $/ 1 / \mathrm{et} / \mathrm{r} /$ finaux ce qui a pour conséquence la convergence formelle, au singulier, entre le type chava(l) [Jav'a] 'cheval' et le type bla [bla] 'blé', ainsi qu'entre le type cube(r) [kyb'e] 'toit' et troupe(l) [trup'e] 'troupeau' (sauf à Salbertrand qui a cubèe [kyb' $\varepsilon$ :]). Le [t] final manifeste aussi une certaine fragilité : à Salbertrand il se maintient dans les monosyllabes et s'amuït dans les polysyllabes ; il s'amuït dans tous les cas à Bardonnèche tandis qu'à Oulx les substantifs et adjectifs monosyllabiques terminés par [t] reçoivent un [ə] final de soutien tandis que le [t] s'amuït dans les polysyllabes : chatë [Jat:ə] 'chat', paqué [pak'e] 'paquet'. Ceci a pour conséquence, localement, la convergence au singulier des types paque(t) [pak'e] 'paquet', cube(r) [kyb'e], trupe(l) [trup'e] (Oulx, Bardonnèche, Rochemolles) ou tcha(t) [tga], bla [bla], tchava(l) [tgav'a] (Bardonnèche).

À Bardonnèche, les pluriels en [s] alternant (type [tat] 'chat', [tas] 'chats') des substantifs monosyllabiques, reçoivent un [ə] de soutien au pluriel et [s] se sonorise : tcha [ $\mathrm{fa}$ ] 'chat', tchasë [ty' azo] 'chats'

À Bardonnèche et Rochemolles on observe la réfection de certains pluriels : bla [bla] 'blé', blau [blau 'blés' (au lieu de blâ [bla:]), sur le modèle de tchavá [tgav'a] 'cheval', tchavau [tav'au] 'chevaux'; paqué [pak'e] 'paquet', paquiau [pakj'au] 'paquets' (au lieu de paques [pak'es]) et cubé [kyb'e] 'toit', cubiau [kybj'au] 'toits' (au lieu de cubé [kyb'e]) sur le modèle de troupé [trup'e] 'troupeau', troupiau [trupj'au] 'troupeaux'; à Rochemolles : lhinsóo [Kĩs' o:] 'drap', Ihinsòu [Kĩs' ou ] 'draps' (au lieu de Ihinsóo [Kĩs 'o:] invariable).

À Salbertrand, Oulx et Bardonnèche, les pluriels des substantifs en -Vp ou -Vk, (type [sap] 'sapin', [fjok] 'feu') se forment par adjonction d'un suffixe [e] (Oulx et Bardonnèche) ou [i] (Salbertrand) : sap [sap] 'sapin', sape [s'ap:e] - sapi [s'ap:i] 'sapins' (au lieu de sap(s) [sap(s)]); fioc [fjok] 'feu', fioque [f'jok:e] - fioqui [f' jok:i] 'feux' (au lieu de fioc(s) [fjok(s)]). À Oulx et Bardonnèche, on a également, fole [f'ol:e] 'fous' au lieu de fòu [foun] et à Oulx, velhe [v'eK:e] 'vieux' (pl.) au lieu de viòu [vjoun].

\footnotetext{
${ }^{19}$ D'après un enquête menée par Lucie Amaro (Université de Lyon II) dans le cadre d'une thèse en cours sur le parler d'Usseaux, localité située à $9 \mathrm{~km}$ de Pragela, et d'après le témoignage de Franc Bronzat.
} 
À Salbertrand, Oulx et Rochemolles, certains noms issus de la première déclinaison latine présentent une alternance vocalique à la tonique : lausë [1'aưzə] 'lause', lòusa [1'ounza] 'lauses' (Salbertrand); mountanhë, [munt'an:ə] 'montagne', mountonha [munt'œn:a] 'montagnes'(Oulx); faitë [fajt $\left.{ }^{(\vartheta)}\right]$ 'faite', fêita [f'cjta] 'faites' (Rochemolles). L'affaiblissement de la tonique est dû à l'attraction exercée par le poids de la voyelle finale, longue ou anciennement longue (qui s'oppose à la voyelle ultra-brève du singulier), ce qui crée une configuration accentuelle «floue», mais on n'observe pas, comme dans certains parlers limousins un véritable déplacement de l'accent tonique sur la finale.

\subsection{Synthèse}

Dans les parlers les plus archaïsants (Champlas, Pragelà, Fénestrelle) les formes du singulier et du pluriel restent assez proches des formes prototypiques telles qu'on peut les reconstituer et dont peuvent être dérivées l'ensemble des formes non innovantes des différents parlers :

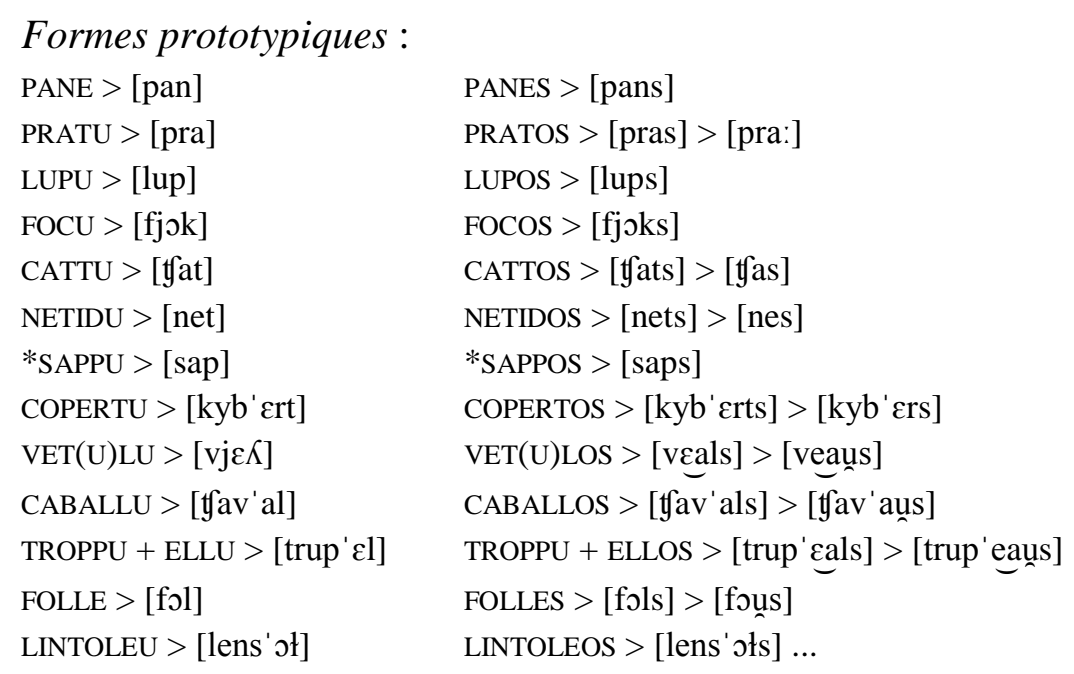

Les parlers de la Haute Vallées du Cluson et de la Haute Vallée de Suse sont représentatifs des différents stades d'évolution de l'occitan parlé dans cette partie des Alpes occitanes, tel

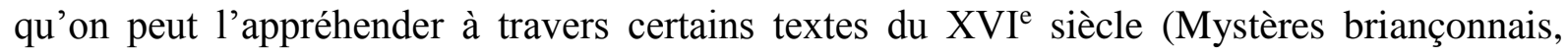
textes juridico-administratifs ; cf. Sibille 2003). Comme souvent, mais sans doute ici plus qu'ailleurs, la diachronie peut se lire dans la synchronie.

Les procédés de marquage du pluriel utilisés par l'ensemble de parlers étudiés sont les suivants :

- Marquage par le seul déterminant (substantifs et adjectifs invariables).

- Suffixe vocalique alternant.

- Allongement vocalique.

- Flexion interne (modification du radical).

À ces quatre procédés il faut ajouter, dans les parlers de Champlas-du-Col, de Pragelà et de Fénestrelle, le marquage du pluriel par -/s/ agglutiné à la forme du singulier et à Salbertrand, 
Oulx et Rochemolles, dans un nombre limités de substantifs, l'apophonie redondante avec le suffixe vocalique alternant ${ }^{20}$.

\section{Conclusion}

Des systèmes complexes de marquage du pluriel existent dans d'autres variétés nord occitanes : alpines, auvergnates, limousines, mais pas dans les parlers en usage dans les autres vallées occitanophones d'Italie ${ }^{21}$; dans ces parlers, seuls les substantifs et adjectifs féminin issus de la $1^{\text {ère }}$ déclinaison latine sont fléchis en nombre: [v'atfo] 'vache', pl. [v'atfes] ou [v'atfa]; les autres substantifs et adjectifs sont invariables (Sibille 2009). Parmi les dialectes gallo-italiques, le piémontais, qui se trouve en contact direct avec les parlers occitans dont il est question dans cet article, a également un système de marquage du pluriel relativement simple : les noms et adjectifs issus de la première déclinaison latine ont un singulier en -[a] et un pluriel en -[e] : [b'uta] 'bouteille', [b'ute] 'bouteilles'; les noms et adjectifs terminés par -[1] ont un pluriel en [j] : [dzurnal] 'journal', [dzurnaj] 'journaux'; les autres substantifs et adjectifs sont invariables (à quelques exceptions près comme [n'ostr] 'nôtre', [n'ostri] 'nôtres').

En revanche, d'autres dialectes gallo-italiques ont des systèmes plus complexes faisant appel à différents procédés ${ }^{22}$ tels que :

- L'apophonie ${ }^{23}$ : [gat] 'chat', [get] 'chats'; [mes] 'mois' (s.), [mis] 'mois' (pl.) ; [ort] ‘jardin', [urt] ‘jardins' (romagnol ; Schürr 1919, Gregor 1971); [bjand] 'blond', [bjond] ‘blonds’ (émilien, Bologne ; Gaudenzi 1889, Mainoldi 1950, Rizzi 1984);

- L'allongement vocalique: [pe] 'pied', [pe:] 'pieds' (émilien, Reggio Emilia ; Bellocchi 1999) ;

- La soustraction, combinée ou non avec l'allongement vocalique et/ou l'apophonie : [dona] 'femme', [don] 'femmes' ; [nime:1] 'cochon', [nime:] 'cochons' (émilien, Reggio Emilia) ; [fjo:l] 'fils' (s.) ; [fju:] 'fils' (pl.) ; [martcl] 'marteau', [marti:] 'marteaux' (émilien, Bologne) ; - la palatalisation de la consonne finale de la base : [gat] 'chat', [gat]] 'chats' ; ['azen] 'âne', ['azen] 'ânes' ; [ka'al] 'cheval', [ka'aj] 'chevaux' (lombard, Brescia ; Bonfadini 1989);

- La suffixation avec effet de métaphonie : [sek] 'sec', [siki] 'secs'; [lu:b] 'loup', [1'ybi] 'loups' ; [ffop] ‘boiteux' (s.), [ţœi] ‘boiteux' (pl.) (ligurien alpin ; Dalbera 1994, 195)...

La problématique du marquage du nombre dans les variétés romanes vernaculaires, notamment nord-occitanes et gallo-italiques, mobilise plusieurs champs disciplinaires: phonétique, phonologie, morphosyntaxe. Ainsi que le souligne Floricic 2010, cette problématique n'a pas, jusqu'à présent - en particulier en domaine occitan - été étudiée avec

\footnotetext{
${ }^{20}$ Ce phénomène ne saurait être qualifié de métaphonie, puisque le timbre de la voyelle tonique ne se rapproche pas de celui de la finale, mais, au contraire, s'en éloigne.

${ }^{21} \mathrm{Du}$ Moyen Cluson, au Nord, à la Haute Vallée du Tanaro, dans la province de Cuneo, au sud.

22 À la différence de l'occitan (si l'on excepte les vallées occitanophones d'Italie situées au sud du Haut Cluson, voir Sibille 2009), dans les dialectes gallo-italiques, les formes du masculin pluriel proviennent de formes en - $i$, comme dans l'ensemble la Romania orientale, bien que ces dialectes aient conservé des traces de morphologie sigmatique dans la flexion verbale. On voit que dans les deux cas, la lénition de la marque du pluriel ( $-s$ dans un cas, $-i$ dans l'autre) aboutit, dans certaines variétés, à des systèmes complexes de marquage du pluriel).

${ }^{23}$ Il s'agit, dans la plupart des cas, d'une apophonie provenant d'une ancienne métaphonie : [g'ati] $>$ [g'cti] > [get], ou [m' ezi] $>$ [m'izi] $>$ [mis].
} 
toute l'attention qu'elle mérite. L'établissement d'une typologie des différents systèmes constituerait un point de départ à des recherches permettant de mieux cerner les dynamiques évolutives dont ces systèmes sont l'aboutissement, et ainsi de mieux comprendre la façon dont interagissent les contraintes phonétiques, phonologiques, morphologiques et syntaxiques.

\section{Bibliographie}

Baccon-Bouvet, Clelia, A l'umbra du cluchî. Salbertrand: patuà e vita locale attraverso i tempi, Torino, Valados Usitanos, 1987.

Barra-Jover, Mario, L'évolution des marques du pluriel nominal roman à la lumière de l'occitan, in: Barra-Jover et al. (2012), 201-216.

Barra-Jover, Mario, et al. (edd), Etudes de linguistique gallo-romane, Saint-Denis, Presses Universitaires de Vincenne, 2012.

Bellocchi, Ugo, Il volgare reggiano. Alle soglie del terzo millennio, Albinea (RE), Tecnograf, 1999.

Berton, Alex/Castagno, Ines/Guiot, Renzo, Prontuario morfologico della parlata occitano-provenzale alpina di Pragelato, Pinerolo, Associazione culturale La Valaddo/Alzani, 2003.

Bonfadini, Giovanni, Caratteristiche e varietà del dialetto bresciano, Atlante Bresciano 21 (1989), 13-25.

Bourlot, Renzo/Martin, Mauro, Prontuario morfologico della parlata occitano-provenzale alpina di Fenestrelle e Mentoules, Pinerolo, Associazione culturale La Valaddo/Alzani, 2007.

Bronzat, Franco, Problemi di interazione linguistica nell'area tra Saluzzo e Pinerolo, tesi de laurea, Università di Torino, 2000.

Calvet, Maurice, Le système phonétique et phonologique du parler provençal de Saint-Victor-en-Vivarais, dégagé sur la base de données instrumentales, Grenoble, Faculté des lettres et sciences de Grenoble, 1969.

Castagno, Ines, et al., Prontuario morfologico della parlata occitano-provenzale alpina di Champlas Janvier et du Col, Pinerolo, Associazione culturale La Valaddo/Alzani, 2003.

Dalbera, Jean-Philippe, Les parlers des Alpes-Maritimes. Etude comparative, essai de reconstruction, London, AIEO/University of London, 1994.

Floricic, Franck, Remarques sur le marquage du nombre dans le parler occitan de Veyrine-de-Vergt, in: id. (ed.) Essais de typologie et de linguistique générale. Mélanges offerts à Denis Creissels, Lyon, ENS Éditions, 2010, 417-433.

Floricic, Franck/Molinu, Lucia, L'Italie et ses dialectes, Lalies 28 (2008), 7-107.

Gaudenzi, Augusto, I suoni, le forme e le parole dell'odierno dialetto della città di Bologna, Torino, Loescher, 1889 (réimpression: Arnaldo Forni, Bologne, 1989).

Gleise-Bellet, Augusta, Appunti morfologici della parlata occitano alpina di Bardonecchia, Oulx, Comunità montana Alta Valle Susa, 2003.

Gregor, Douglas, Romagnol. Language and Literature, Cambridge, The Oelander Press, 1971.

Grosso, Michela, Grammatica essenziale della lingua piemontese, Torino, Libreria piemontese, 2000.

Hirsch, Ernst, Provenzalische Mundarttexte aus Piemont, Tübingen, Niemeyer, 1978.

Jayme, Giovanna, Prontuario morfologico della parlata occitano-provenzale alpina di Oulx, Pinerolo, Associazione culturale La Valaddo - Alzani, 2003.

L'occitano dell'alta Val Pellice, studio morfologico, [S.1.], Provincia di Torino/Comunità Montana Val Pellice/Società di Studi Valdesi, 2007.

Mainoldi, Pietro, Manuale dell'odierno dialetto bolognese. Suoni e segni, grammatica, vocabolario, Bologna, Società tipografica Mareggiani, 1950.

Martin, Jean-Baptiste, Le pronom personnel de la $3^{e}$ personne en francoprovençal central (formes et structures), Travaux de linguistique et de littérature, XI, 1 (1974), 85-116.

Masset, Angelo, Grammatica del patois provenzale di Rochemolles, Borgone di Susa, Melli, 1997.

Perron, Piero, Grammatica del patoua di Jouvenceaux, Torino, Valados Usitanos, 1984.

Pons, Teofilo/Genre, Arturo, Prontuario morfologico del dialetto occitano-provenzale alpino della Val Germanasca, Pinerolo, 2003.

Rizzi, Elena, L'apofonia nel dialetto di Bologna. Una proposta di analisi morfofonemica, Rivista italiana di dialettologia 8 (1984), 91-108. 
Sauzet, Patrick, Los morfèmas de plural nominal a Sant Julian de Cremsa: [-w] e lo ton bas, in: Actes du $9^{e}$ congrès de l'AIEO, vol. 2, Aachen, Shaker, 2011, vol. 2, 827-842.

Schür, Friedrich, Romagnolische Dialektstudien, 2 vol., Vienne, Kaiserliche Akademie der Wissenschaften in Wien, 1918-1919.

Sibille, Jean, La Passion de saint André, drame religieux de 1512 en occitan briançonnais. Édition critique, étude linguistique comparée, Thèse de l'Université de Lyon II, 2003.

Sibille, Jean, L'évolution des parlers occitans du Briançonnais, ou comment la diachronie se déploie dans l'espace, Cahiers de grammaire 29 (2004), 121-141.

Sibille, Jean, Les formes en $-i$ issues du nominatif pluriel de la $2^{\text {ème }}$ déclinaison latine, en occitan. Essai d'approche panchronique, in: Fréchet, Claudine (ed), Langues et cultures de France et d'ailleurs. Hommage à Jean-Baptiste Martin, Lyon, Presses Universitaires de Lyon, 2009, 233-250.

Sibille, Jean, La marca del numero nella parlata occitana di Sénaillac-Lauzès (Francia), Rivista italiana di dialettologia 35 (2011), sous presse.

Sibille, Jean, Parentés génétiques affinités aréales et évolutions spécifiques dans les parlers occitans des vallées d'Oulx et du Haut-Cluson (Italie), in: Barra-Jover et al. (2012), 67-83.

Talmon, Alberto, Saggio sul dialetto di Pragelato, Archivio glottologico italiano 17 (1914), 1-101.

Telmon, Tullio/Canobbio, Sabina, Atlante Linguistico e Etnografico del Piemonte Occidentale, Pavone Canavese, Priuli \& Verlucca, 2004ss. (en cours de publication).

Vayr, Enzo/Telmon, Tullio, Appunti morfologici della parlata francoprovenzale di Giaglione, Oulx, Comunità montana Alta Valle Susa, 2004.

Vignetta, Andrea, Patuà. Grammatica del dialetto provenzale-alpino della medio-alta Val Chisone, Pinerolo, Alzani, 1981.

\section{Annexe 1 : Conventions graphiques.}

Voyelles:

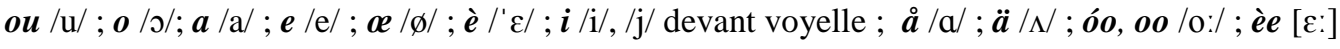

$\boldsymbol{u} / \mathrm{y} /$, sauf s'il est $2^{\text {ème }}$ élément d'une diphtongue descendante.

$\ddot{\boldsymbol{e}}[$ [ə] faiblement articulé ou totalement « muet »;

Les voyelle nasales sont notées par un $\boldsymbol{n}$ après la voyelle oun $/ \tilde{\mathrm{u}} /$; an $/ \tilde{a} /$ etc. ;

L'accent circonflexe note une voyelle longue : $\hat{\boldsymbol{\imath}} / \mathrm{i}: / ; \hat{\boldsymbol{a}} / \mathrm{a}:$; $\hat{\boldsymbol{o}} / \mathrm{\jmath} / \mathrm{l}$ etc.

Diphtongues :

$\boldsymbol{a u} / \mathrm{au} /$; òu /oun/ ; $\boldsymbol{e i} / \mathrm{ei} /$; $\boldsymbol{\alpha} i / \varnothing \mathrm{i} / \ldots$

Consonnes :

$\boldsymbol{p}, \boldsymbol{t}, \boldsymbol{b}, \boldsymbol{f}, \boldsymbol{v}, \boldsymbol{l}, \boldsymbol{m}, \boldsymbol{n}:$ même valeur qu'en API ;

$\boldsymbol{c h} / \mathrm{f} / \boldsymbol{j} / \mathrm{J} / \boldsymbol{l} \boldsymbol{h} / \mathrm{K} / \boldsymbol{n h} / \mathrm{n} / ; \boldsymbol{t}$ ou $\boldsymbol{t} \boldsymbol{c h} / \mathrm{g} / ; \boldsymbol{d j} / \mathrm{d} / ; \boldsymbol{t} / \mathrm{t} /(l$ vélaire $)$

$\boldsymbol{c}$, ou $\boldsymbol{q u}+e, i / \mathrm{k} / ;, \boldsymbol{g}$, ou $\boldsymbol{g} \boldsymbol{u}+e, i / \mathrm{g} / ;$

$\boldsymbol{s} \boldsymbol{s}$ intervocalique et $\boldsymbol{s}$ non intervocalique /s/ ;

$\boldsymbol{s}$ intervocalique, ou final en liaison devant voyelle /z/ ;

$\boldsymbol{r}$ intervocalique $/ \mathrm{r} / ; \boldsymbol{r} \boldsymbol{r}$ intervoc. et $\boldsymbol{r}$ non intervoc. $/ \mathrm{r} /$

Accent tonique :

Les mots terminés par une voyelle sans accent graphique sont paroxytons : vacho /'va So/ 'vache', pòure /'powre/ 'pauvre'... les autres mots sont oxytons : bachas /baf'as/ 'vasque', anâ /an'a:/ 'aller', bichit /bif' it/ 'petit'.

Lorsque cela est nécessaire, l'accent tonique est indiqué par un accent aigu, ex : aná 'allé', joúvan 'jeune'. 
Annexe 2 : Carte

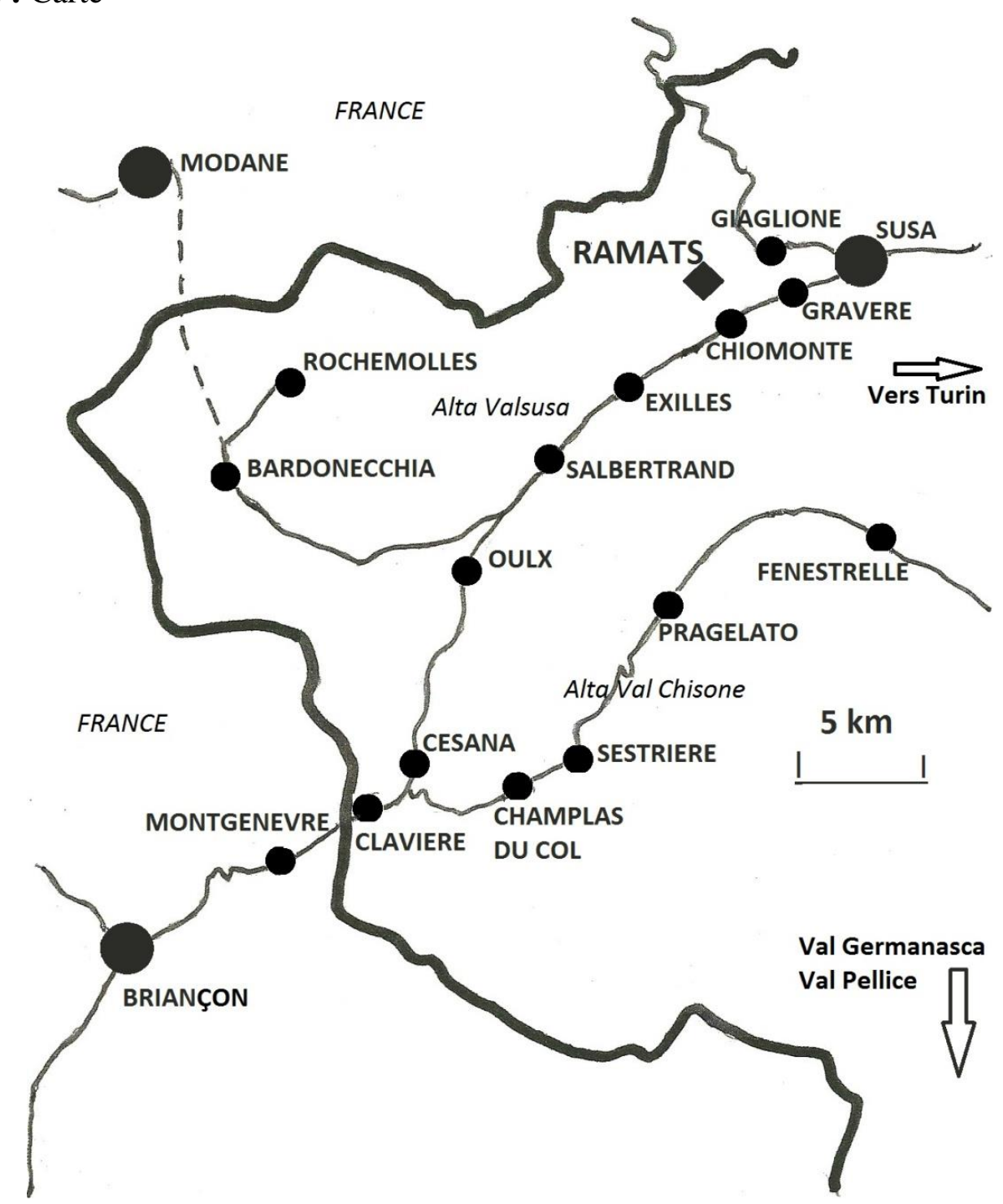

Frontière franco-italienne.

Routes. - - - Tunnel.

\section{Annexe 3}

Distances par la route, en km, entre les différentes localités

\begin{tabular}{|l|c|c|c|c|c|c|c|c|}
\hline & Fénestrel. & Pragelà & Champlas & Ramats & Salbertr. & Oulx & Bardonn. & Rochem. \\
\hline Fénestrelle & - & 12 & 26 & 61 & 50 & 44 & 57 & 64 \\
\hline Pragelà & 12 & - & 14 & 49 & 38 & 32 & 45 & 52 \\
\hline Champlas & 26 & 14 & - & 35 & 24 & 18 & 31 & 38 \\
\hline Ramats & 61 & 49 & 35 & - & 12 & 18 & 31 & 38 \\
\hline Salbertrand & 50 & 38 & 24 & 12 & - & 6 & 19 & 26 \\
\hline Oulx & 44 & 32 & 18 & 18 & 6 & - & 13 & 20 \\
\hline Bardonnèche & 57 & 45 & 31 & 31 & 19 & 13 & - & 7 \\
\hline Rochemolles & 64 & 52 & 38 & 38 & 26 & 20 & 7 & - \\
\hline
\end{tabular}

